\title{
Ein altuigurisches Dharmaśarīrasūtra nach einer chinesischen Version
}

\author{
Peter ZIEME*
}

(Berlin)

\begin{abstract}
Abstrakt: Das altuigurische Fragment U 4830 (T II Y 6) der Berliner Turfan-Sammlung wird hier zusammen mit der originalen chinesischen Passage des Dharmaśarīrasūtra (Foshuo) fashen jing (佛説)法身經(T.XVII.0766.0699b18-700c08) ediert und verglichen. Bilinguale sanskritische/altuigurische Fragmente Textes waren bereits bekannt. Das hier veröffentlichte Bruchstück bezeugt einmal mehr, daß bei den uigurischen Buddhisten ein Interesse für das Sūtra oder vielleicht sogar ganz allgemein für dogmatische Begriffsreihen bestand.
\end{abstract}

Schlüsselwörter: Altuigurisch, bilinguale Texte, buddhistische Begriffsreihen, Dharmaśarīrasūtra.

\section{Abstract: An Old Uyghur Dharmaśarīrasūtra According to a Chinese Version}

The Old Uyghur fragment U 4830 (T II Y 6) of the Berlin Turfan Collection is edited and compared here together with the original Chinese passage of the Dharmaśarīrasūtra (Foshuo) fashen jing (佛 説)法身經 (T.XVII.0766.0699b18-700c08). Bilingual Sanskrit/Old Uyghur fragments of the text were already known. The piece published here testifies once more that Uyghur Buddhists studied on this sūtra or, more generally, on dogmatic terms.

Key Words: Old Uyghur, bilingual texts, Dharmaśarīrasūtra, Buddhist terms.

* Prof.Dr., Berlin-Brandenburgische Akademie der Wissenschaften, ziemepet@gmail.com; ORCID: 0000-0002-8090-7707.

Received: 16.06.2021; Accepted: 29.06.2021;

Published: 30.06 .2021 


\section{Einleitung:}

Kurz nach den großen Entdeckungen von Textfunden in den Oasen des Tarimbeckens hatte 1904 H. Stönner einen Sanskrittext ediert, dessen Titel Dharmaśarīrasūtra ist", den er als "Sūtra, das die Grundlage der Religion enthält" übersetzte. Das weist schon sehr deutlich auf den Inhalt des Sūtras hin: dogmatische Begriffe, die aufgezählt und erläutert werden. ${ }^{2}$ Sanskritfragmente aus St. Petersburg wurden von G.M. Bongard-Levin \& M.I. VorobyovaDesjatovskaja ediert ${ }^{3}$.

Chotan-sakische Fragmente haben G.M. Bongard-Levin \& E.N. Tyomkin veröffentlicht. ${ }^{4}$ Eine Brāhmī-Handschrift von sanskrituigurischen bilingualen Fragmenten ${ }^{5}$ bezeugt, daß uigurische Kreise eng vertraut waren mit diesem Sūtra und vergleichbaren Texten. ${ }^{6}$

Als Gegenpol kann das hier vorgestellte Fragment gelten, denn sein Ausgangspunkt ist zweifelsfrei eine chinesische Version des Textes, und zwar vermutlich T766. Diese chinesische Version des Dharmaśarīrasūtra lautet (Foshuo) fashen jing (佛説)法身經 (T.XVII.0766.0699b18-700c08). Fan \& Peng \& Wang haben die

Stönner 1904, 1282.

Herrn Klaus Wille verdanke ich Hinweise auf weitere Sanskritfragmente. Vgl. Wille 2014, 187, 203 (für T766 sind die vermuteten Parallelfragmente besonders interessant); Hartmann \& Wille 2014a (Otani-Sammlung), 251 (Bezeklik 80 TBI 764+772; identifiziert von D. Maue); Hartmann \& Wille 2014b, 215; Wille 2015 (London Or.15015/301. Ferner fügt er hinzu: “ $\mathrm{Zu}$ SHT 1689 ist noch ein neues Fragment dazugekommen: SHT VII $1689+$ XII 6991 (belongs to 1689b)."

3 Bongard-Levin \& Vorobyova-Desjatovskaja 1985, 65-76.

4 Bongard-Levin \& Tyomkin 1969.

5 fol. 79: Maue 2008, foll. 63 und 67: Maue \& Zieme 2012 ; Fan \& Peng \& Wang 2018.

6 An dieser Stelle richte ich ein besonderes Dankeswort an Dieter Maue, der nicht nur die bilingualen Fragmente dieses Sūtras ediert hat, sondern auch meinen hier folgenden Beitrag durch seine Expertise bereichert hat. 
vorangegangenen chinesischen Versionen des Textes ausführlich dokumentiert $^{7}$, sie geben den Titel des von Faxian (法賢, *Dharmabhadra) zur Zeit der Nördlichen Song-Dynastie übersetzten Sūtra T766 in der Rekonstruktion *Dharmakāya-sūtra, nicht zuletzt weil fashen 法身 am ehesten dharmakāya wiedergibt. Allerdings trifft ihr Argument, daß der Sanskrit-Titel fehle, nicht zu, denn der ist in dem Berliner Fragment, das 1904 H. Stönner ediert hatte, als dharmaśarīrasütra belegt ${ }^{8}$ sowie auch in der chotansakischen Version, die Bongard-Levin \& Tyomkin 1969 publiziert haben ${ }^{9}$.

Das aus Yarchoto stammende Fragment eines altuigurischen Faltbuches, das in KOHD Digital beschrieben ${ }^{10}$ und dessen Text in einem Aufsatz über Kanișka zitiert wurde ${ }^{11}$, erweist sich als eine altuigurische Übersetzung der chinesischen Version des Dharmaśarīrasūtras (T766). Der altuigurische Titel ist nicht überliefert, doch der Terminus d(a)rmaš(a)rir nomlug ät'öz "dharmaśarīra (=) Lehr-Körper" ist in der altuigurischen Fassung des Cibei daochang chanfa 慈悲道場懺法 (altuig. Kšanti kllguluk nom bitig) die Übersetzung von fashen 法身. ${ }^{12}$

Daraus kann abgeleitet werden, daß für das Sūtra oder vielleicht sogar ganz allgemein für dogmatische Begriffsreihen bei den uigurischen Buddhisten ein Interesse bestand, und zwar in beiden

Fan \& Peng \& Wang 2018, 287.

8 Stönner 1904, 1282. Herr Klaus Wille teilte mir mit, daß auch der ursprünglich in SHT IX S. 424 angenommene Titel (dha)[rma](kāyā)[s] nach Hori 2005, S. 92 doch eher zu (dha)[rma](śa)[rīr](a)sūtram ergänzt werden kann, worauf in SHT X S. 432f dann hingewiesen wurde. Der Titel des Sūtra ist auch in dem Pelliot-Fragment (Pelliot divers D.A. G) linkes Fragment, Zeile Af-g erhalten: f kuruta bhāṣișy. [dharma] .. g rīraṃ nāma dharmaparyah yah.

9 Bongard-Levin \& Tyomkin 1969, p. 272: 7A1 dharmaśśarīrä sūträ.

10 Vgl. KOHD Digital (Beschreibung: S.-Chr. Raschmann).

11 Zieme 2018, 179-180.

12 BT 25, ZZ. 3712-3713 (HWAU 245a). 
Traditionslinien, in dem vom Sanskrit geprägten Schrifttum wie auch in der vom chinesischen Buddhismus geleiteten Sphäre. Für die inhaltliche Einordnung des Textes möge an dieser Stelle ein Zitat aus der Abhandlung über die Trikāya-Lehre von Jens Wilkens genügen: "Im Laufe der Zeit entwickelte sich dann allerdings eine Doktrin, die eine Dichotomie von dhammakāya und rūpakāya postulierte, wobei in ersterem eine Repräsentation der buddhistischen Schriften zu sehen ist, während in letzterem eine Bezeichnung für die physische Existenz des Buddha, dem die 32 Schönheitsmerkmale eines großen Mannes und die 80 Nebenmerkmale zukommen und der in den Reliquien manifest ist, erkennbar wird." ${ }^{\prime 13}$ Der hier behandelte Text steht auf der Stufe der Dichotomie, nennt aber nicht die generelle Bezeichnung für den physischen Körper des Buddha rūpakāya, sondern den nirmānakāya = huashen als Gegensatz zum dharmakāya. In der Dogmatik ist jedoch der nirmānakāya neben dem sambhogakāya eine der zwei Seiten des rüpakāya. Es ist bemerkenswert, daß der Uigure nicht den zu erwartenden Begriff öylüg ätöz "rūpakāya"14 verwendet, sondern die Neubildung tugma ätöz, die vielleicht dem erstgenannten Merkmal geschuldet ist, nämlich, daß der Buddha von Mutter und Vater geboren wird, im Altuigurischen ausgedrückt durch "aufgezogen", wie aus dem Textvergleich hervorgeht. Daß von dem Übersetzer die beiden Körper in den Verehrungsteil gestellt werden, ist ein beachtenswertes Charakteristikum dieses Textes, aber derartige "Personifizierungen" sind dem uigurischen Buddhismus auch in anderen Zusammenhängen nicht fremd.

\footnotetext{
BT 21, 15.

4 HWAU 537a. Die zusammengesetzten Fragmente U 5402r + U 5473r erörtern in einem noch nicht identifizierten Text, wie der önsüz ätöz “*ārūpyakāya” zu verstehen ist. In VOHD 13,12 wurden nur die publizierten Rückseiten der Fragmente beschrieben (Nr. 235 und 236).
} 


\section{Das Fragment}

U 4830 (T II Y 6). Es handelt sich um ein handschriftliches Faltbuch mit fünf Zeilen pro 'Seite'. Die Einleitung, die Zeilen 01 bis 06, sind mit roter Tusche geschrieben, ist eine Anrufung des Triratna. Im anschließenden ersten Teil werden die Charakteristika des nirmānakāya des Buddha beschrieben. Der nicht erhaltene zweite Teil, der sicher einen etwas längeren Umfang hatte, war der Definition und Beschreibung des fashen 法身 dharmakāya gewidmet.

\section{Anrufung des Triratna}

01 yükünürmn burhan-ka .. yükünürmn nom 02 -ka .. yükünürmn bursan kuvrag-ka ..

\section{Übersetzung der Triratna-Formel}

Ich verneige mich vor dem Buddha.

Ich verneige mich vor dem dharma.

Ich verneige mich vor dem samgha.

\section{Charakteristika des nirmānakāya}

In der folgenden Tabelle ${ }^{15}$ wird ein Vergleich zwischen der chinesischen Vorlage und der altuigurischen Übersetzung vorgestellt.

\begin{tabular}{|c|c|c|}
\hline & U 4830 & T766 \\
\hline 01 & & $\begin{array}{l}\text { 699b21 爾時世尊於大衆中以微妙音作如是言 } \\
\text { Zu dieser Zeit sprach der Erhabene in der großen } \\
\text { Schar mit feiner Stimme. }\end{array}$ \\
\hline 02 & $\begin{array}{l}03 \text { yükünürmn alku gạ } \\
\text { ögüzdäki kum } 04 \text { sanınča } \\
\text { köni tüz tuymıšlarnıy }\end{array}$ & 諸 ${ }^{699 \mathrm{~b} 22}$ 佛如來 Alle Buddhas sind Tathāgatas, \\
\hline
\end{tabular}

15 Die den chinesischen Syntagmen nachgestellten deutschen Übersetzungen (kursiv) sind nur zur Orientierung gedacht. 


\begin{tabular}{|c|c|c|}
\hline 03 & $\begin{array}{r}\text { iki } 05 \text { [törlüg } \\
\text { ]nin }\end{array}$ & $\begin{array}{l}\text { 有二種身皆具河沙功徳 sie haben zwei Arten } \\
\text { von Körpern, beide haben Vorzüge zahlreich } \\
\text { wie Fluß-Sand. }\end{array}$ \\
\hline 04 & & 何等爲二 Was sind die zwei? \\
\hline 05 & $\begin{array}{l}\text { tugma ät’öz } 06 \text {-lärinä } \\
\text { ymä ... .. } \\
\text { nomlug ät'özlärinä }\end{array}$ & $\begin{array}{l}{ }^{699 b 23} \text { 所謂化身法身 Sie werden genannt } \\
\text { Verwandlungskörper und Dharmakörper. }\end{array}$ \\
\hline 06 & 07 tugma ät'özläri & $\begin{array}{l}\text { 而化身者 Und was den Verwandlungskörper } \\
\text { betrifft, }\end{array}$ \\
\hline 07 & $\begin{array}{l}{[\ddot{o g}] \text { kạ igidmiš } \mathbf{0 8}} \\
\text { balturtmıš }\end{array}$ & $\begin{array}{l}\text { 示從父母所生 ist er angezeigt wie von Vater } \\
\text { und Mutter geboren: }\end{array}$ \\
\hline 08 & $\begin{array}{l}\text { iki kırk ulug äränlär } 09 \text {-nin } \\
\text { lakšanlar } \\
\text { üzä etiglig }\end{array}$ & $\begin{array}{l}\text { 699624 具三十二相 versehen mit den } 32 \\
\text { lakșaṇas, }\end{array}$ \\
\hline 09 & $\begin{array}{l}\text { säkiz } \mathbf{1 0} \text { on nayragın } \\
\text { yaratıglıg .. }\end{array}$ & $\begin{array}{l}\text { 八十種好莊嚴 geschmückt mit den } 80 \\
\text { anuvyañjanas. }\end{array}$ \\
\hline 10 & $\begin{array}{l}\text { [tınlıgları] } 11 \\
\text { ögirtdürdäči .. } \\
\text { köz kačigıg k[utadturdači] }\end{array}$ & $\begin{array}{l}\text { 其身以智慧眼 }{ }^{699 b 25} \text { 普觀衆生 } \\
\text { 智者瞻仰 心生適悦 } \\
\text { Dieser Körper hat Augen der Weisheit, } \\
\text { Universalsicht auf alle Wesen, als Weiser } \\
\text { verehrt er respektvoll, das Hervorbringen des } \\
\text { Sinnes ist ein Vergnügen. }\end{array}$ \\
\hline 11 & & 三業清淨 Rein in den drei Aktivitäten. \\
\hline 12 & $\begin{array}{l}12 \text { birär birärintä } \\
\text { yüz yüz buyan[lar üzä] } 13 \\
\text { tutulmı̌̌ }\end{array}$ & $\begin{array}{l}\text { 699b26一一相好 百福具足 Jede der } \\
\text { Eigenschaften ist vollkommen mit hundert } \\
\text { Tugenden. } \\
\text { 如是莊嚴 百千福聚 Ebenso sind die } \\
\text { Verzierungen hunderttausende angehäufte } \\
\text { Segnungen. }\end{array}$ \\
\hline 13 & $\begin{array}{l}\text { [ulug] äränlärnin } \\
\text { b(ä)lgülärinä } 14 \text { tükällig .. }\end{array}$ & $\begin{array}{l}\text { 大 }^{699 \mathrm{~b} 27} \text { 丈夫相 Die Merkmale eines großen } \\
\text { Mannes sind }\end{array}$ \\
\hline 14 & $\begin{array}{l}\text { öy ügmäkdä tutulmıš on } \mathbf{1 5} \\
\text { küčlärnị .. }\end{array}$ & $\begin{array}{l}\text { 皆色蘊攝 } \text { 又復具足十力 alle subsumiert in } \\
\text { der rüpa-Anhäufung sowie auch vollkommen } \\
\text { mit den } 10 \text { Kräften, }\end{array}$ \\
\hline 15 & $\begin{array}{l}\text { tört törlüg korkınčsız } 16 \text { - } \\
\text { larnıy .. }\end{array}$ & 四無所 ${ }^{699 b 28}$ 畏 in den 4 Furchtlosigkeiten, \\
\hline 16 & & 三不空法. in den 3 nicht-leeren dharmas, \\
\hline
\end{tabular}




\begin{tabular}{|c|c|c|}
\hline 17 & $\begin{array}{l}\text { üč törlüg ymä äyäyük ög } 17 \\
\text { turuglarnıy .. }\end{array}$ & 三念住法 in den 3 dharmas der Sinn-Ruhe, \\
\hline 18 & $\begin{array}{l}\text { üč törlüg küzädgülük } \mathbf{1 8} \text { - } \\
\text { süzlärnị [..] }\end{array}$ & 三不護法 in den 3 dharmas des Nicht-Hütens, \\
\hline 19 & & $\begin{array}{l}\text { 四無量 }{ }^{699 \mathrm{~b} 29} \text { 法 (= 心) in den vier } \\
\text { Unermeßlichen Sinn-zuständen). }\end{array}$ \\
\hline 20 & $\begin{array}{l}\text { ulug y(a)rlıkančučı } \\
\text { köyülnün .. }\end{array}$ & s. 19 \\
\hline 21 & $\begin{array}{l}19 \text { ulug täy könülnüy ymä .. } \\
\text { idišsi bolmiš .. }\end{array}$ & s. 19 \\
\hline 22 & 20 birär birär yüzägülärindä & $\begin{array}{l}\text { 具大丈夫 Der vollkommene große Mann, } \\
\text { 一一最勝 ein jeder ein Jina, }\end{array}$ \\
\hline 23 & $\begin{array}{l}\text { naray[an küčläri] } \mathbf{2 1} \\
\text { tutulmıš .... }\end{array}$ & 那羅延力 hat die Kraft Nārāyaṇas. \\
\hline 24 & muntada ulatı kavira & 如是略説 Und so kurz gesagt: \\
\hline 25 & $\begin{array}{l}\text { ädgülüg } 22 \text { adrokları üzä .. } \\
\text { tükälliglärnin .. }\end{array}$ & s. $29+30$ \\
\hline 26 & $\begin{array}{l}23 \text { ančulayu } \\
\text { käl[miš]lärnin .. }\end{array}$ & 699c01 如來 [1] Der Tathāgatas, \\
\hline 27 & ayagka tägimlig 24 -lärnī .. & 應供[2] derArhats, \\
\hline 28 & köni tüz tuymıšlarnı̀ .. & $\begin{array}{l}\text { 正等正覺 [3] der aufrichtigen } \\
\text { Samyaksambuddhas, }\end{array}$ \\
\hline 29 & s. $25+26$ & $\begin{array}{l}\text { 莊嚴功徳 der dieTugenden der Verzierungen } \\
\text { Habenden, }\end{array}$ \\
\hline 30 & s. $26+26$ & $\begin{array}{l}\text { 具足圓滿 der mit den Vollkommenheiten } \\
\text { Versehenen }\end{array}$ \\
\hline 31 & bo 25 ärür tugma ät'özläri .. & 是 ${ }^{699 \mathrm{c} 02}$ 名化身 nirmānakāya ist so. \\
\hline 32 & & $\begin{array}{l}\text { 又復諸佛如來應供正等正覺所有 Und so } \\
\text { aller Buddhas, Tathāgatas, Arhats, } \\
\text { Samyaksambuddhas }\end{array}$ \\
\hline 33 & nomlug ät'özläri & 699c03 法身 Dharma-Körper (...) \\
\hline
\end{tabular}

Da in der uigurischen Übersetzung der Abschnitte 02 bis 05 noch die vom Anfang des Textes gewohnte Verehrungsformel fortgeführt wird, hat der Übersetzer 01 nicht übersetzt. Der chinesische Text besagt, daß der Buddha eine Rede an die Gemeinde hält, in 
der die Charakteristika der beiden Körper, nirmānakāya und dharmakāya, vorgestellt werden. Erst mit Abschnitt 06 richtet der uigurische Übersetzer seinen Text so ein, wie es die chinesische Vorlag wohl erfordert.

Übersetzung

(02-05) Ich verneige mich vor den zwei [Arten von ], den Geburtskörpern und den Dharma-Körpern aller wahrhaft und gleichmäßig Erleuchteten, die zahlreich sind wie Sandkörner im Ganges-Fluß.

(06) Geburtskörper haben sie, (07) weil sie von Mutter und Vater aufgezogen werden, (08) geschmückt mit den 32 lakșanas der großen Männer, (09) ausgestattet mit den 80 anuvyañjanas, (10) [die Lebewesen] erfreuend, den Augen-[Sinn beglückend]. (12) In je einem der (Vorzüge) sind Hunderte von punyas erfaßt, (13) vollkommen mit den Merkmalen des [großen] Mannes.

(21) Sie sind Gefäße der

(14) zehn Kräfte, die in der rūpa-Anhäufung enthalten sind,

(15) der viererlei Furchtlosigkeiten,

(17) der dreierlei spezifischen Sinn-Zustände,

(18) der dreierlei Nicht zu Hütenden,

(20) der großen Barmherzigkeit,

(21) der großen Gleichmut.

(22-23) In jedem der Glieder sind Nārāy[aṇa-Kräfte] enthalten.

(24) Und so weiter, kurz:

(31) Darin bestehen die Geburtskörper

(25) derjenigen, die an Gutem und Vorzügen vollkommen sind,

(26) der So-Gekommenen,

(27) der Verehrungswürdigen,

(28) der wahrhaft und gleichmäßig Erleuchteten.

(33) Ihre Dharma-Körper [sind folgende: ...] 


\section{Anmerkungen}

(02) Die Mengenangabe "wie Sand(körner) im Fluß (Ganges)" ist im chinesischen Text auf die Vorzüge bezogen: hesha gongde, doch der Uigure nimmt dies als Attribut zu den wahrhaft und gleichmäßig Erleuchteten.

(03) Es ist schwierig, die Lücke auszufüllen, doch sicher dürfte törlüg nach chin. 種 “Art” sein. Dem Chin. zufolge könnte man anschließend 身 “Körper” annehmen, doch bleibt dies zweifelhaft.

(05) tugma ät'öz für "Erscheinungskörper" als Übersetzung von huashen 化身 ist nur hier und in U 2972 belegt (vgl. Zieme 2018, 179), viel üblicher ist bälgürtmä ätöz. Allerdings wird der "Geburtskörper” shengshen 生身 in der Regel vom huashen 化身 unterschieden (vgl. DDB).

(13) Die Ergänzung folgt chin. da zhangfu 大丈夫 = skt. mahāpuruṣa "großer Mann", Epithet (> Bezeichnung) eines künftigen Cakravartin oder eines Buddha (cf. SWTF III, 343a). Die altuig. Entsprechung äränlär "Männer" steht hier im Plural, ähnlich auch in der Trikāya-Lehre des Goldglanz-Sūtras: ulug

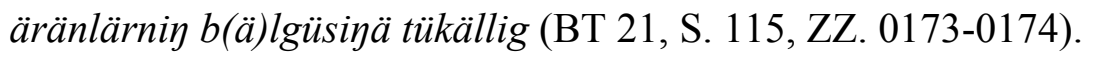

(18) Nach der Erleuchtung müssen die Buddhas sich nicht mehr vor falschen Aktivitäten in den drei Bereichen (Körper, Rede, Sinn) hüten (vgl. DDB; skt. trīny arakṣyāṇi).

(19) Dieser Terminus ist uig. nur indirekt wiedergegeben, indem nicht der Oberbegriff genannt wird, sondern zwei der vier unermeßlichen Sinn-Zustände: (20) und (21).

(20) "Große Barmherzigkeit" = skt. mahākarun̄a ist im chinesischen Text an dieser Stelle nicht erwähnt. Es ist das zweite Element der vier unermeßlichen Sinn-Zustände (catvāri-apramānāni, DDB). 
(21) täy köyül "Gleichmut" (HWAU 694a) = skt. upekșa $\bar{a}^{16}$ ist im chinesischen Text an dieser Stelle nicht erwähnt. Gleichmut ist nach Liebe, Barmherzigkeit und Mitfreude das vierte Element der vier unermeßlichen Sinn-Zustände (catvāri-apramānāṇi; DDB). Der Berliner Text U 1890 recto 13-14 listet die 4 Bestandteile auf: maitri [.. ka]run .. mudit .. upekšs .. bo tört apramani "maitrī, karuṇā, muditā, upekșā - diese vier apramānāṇi”. Die Aufzählung liegt des weiteren auch in TT VIII F 10 sowie in Maue 2015, 115v3 vor.

(21) idiš “Gefäß”. Für diese Aussage gibt es keine Vorlage, doch dürfte gemeint sein, daß der rūpakāya eben ein Gefäß seines dharmakāya ist, wofür eine ausdrückliche Aussage K. Kitsudō nachweisen konnte: T.III.0156.157a06-07 答日. 色身是法身器故 "Die Antwort lautet: Weil der rūpakāya das Gefäß des dharmakāya ist."

(22) Die erste chin. Gruppe 具大丈夫 hat kein Pendant. Für - ist die Übersetzung “je ein” einleuchtend, doch es bleibt völlig unklar, warum jina (zuisheng 最勝) hier durch yüzägülärindä “in den Gliedern" übersetzt wurde.

(26-28) Diese Abschnitte entsprechen den ersten drei der 10 Standardepitheta des Buddha, vgl. zu den einzelnen Benennungen: 如來, vgl. Nattier 2003, 210 Tathāgata a; 應供, vgl. Nattier 2003, 215-216 Arhat c; 正等正覺, vgl. Nattier 2003, 220 Samyaksambuddha $\mathrm{b}$ (ohne vorangestelltes 正).

(32-33) Ab hier beginnt dann der nächste Abschnitt des Textes, dessen altuigurische Fassung noch unbekannt ist.

\footnotetext{
${ }^{16}$ Auch der indische Terminus ist belegt, vgl. HWAU 802a.
} 


\section{Literatur}

BT $21=$ Wilkens 2001

BT $25=$ Wilkens 2007

HWAU = Wilkens 2021

SHT VII $=$ Wille 1995

SHT IX $=$ Wille 2004

SHT X $=$ Wille 2008

SWTF $\quad=$ Sanskrit-Wörterbuch

TT VIII $=$ v. Gabain

VOHD 13, $12=$ Özertural

Bongard-Levin, G.M. \& Tyomkin, E.N. 1969. Fragment of the Saka version of the Dharmaśarīra-sūtra from the N.F. Petrovsky collection. In: Indo-Iranian Journal 11.4 (1969), 269-280.

Bongard-Levin, G.M. \& Vorobyova-Desjatovskaja, M.I. 1985. Pamjatniki indijskoj pis'mennosti iz Central'nogo Azii, vypusk 1. Moskva.

Fan, Jingjing \& Peng, Jinzhang \& Wang, Haiyun. 2018. Three More Leaves of the Sanskrit-Uigur bilingual Dharmaśarīrasūtra in Brāhmī Script. In: Acta Orientalia Academia Scientiarum Hungaricae 71, 285-301.

Gabain, Annemarie von. 1954. Türkische Turfantexte VIII. Abhandlungen der Deutschen Akademie der Wissenschaften zu Berlin Kl. Für Sprachen, Literatur und Kunst Jg. 1952, Nr. 7. Berlin.

Hartmann, Jens-Uwe \& Wille, Klaus. 2014a. Further Collections of Sanskrit Manuscripts from Central Asia. In: From Birch Bark to Digital Data: Recent Advances in Buddhist Manuscript Research Papers Presented at the Conference Indic Buddhist Manuscripts: The State of the Field Stanford, June 15-19 2009. Edited by Paul Harrison and Jens-Uwe Hartmann. Wien, 247-255. 
Hartmann, Jens-Uwe \& Wille, Klaus. 2014b. The Central Asian Sanskrit Fragments in the Pelliot Collection (Paris). In: From Birch Bark to Digital Data: Recent Advances in Buddhist Manuscript Research Papers Presented at the Conference Indic Buddhist Manuscripts: The State of the Field Stanford, June 15-19 2009. Edited by Paul Harrison and Jens-Uwe Hartmann. Wien, 213-222.

Hori, Shin'ichirō. 2005. Additional Notes on the Unidentified Sanskrit Fragments in the Ōtani Collection at Ryūkoku University Library. In: Journal of the International College for Postgraduate Buddhist Studies 9 (2005), 91-97.

Maue, Dieter. 2008. The equanimity of the Tathāgata. Aspects of research into Central Asian Buddhism, ed. by Zieme, Peter. Turnhout (Silk Road Studies XVI), 179-190.

Maue, Dieter. 2015. Alttürkische Handschriften. Teil 19. Dokumente in Brāhmī und tibetischer Schrift, Teil 2, Stuttgart.

Maue, Dieter \& Zieme, Peter. 2012. Two More Leaves of the Dharmaśarīrasūtra in Sanskrit and Uigur. In: Historical and Philological Studies of China's Western Regions (西域歷史 語言研究集刊), 2012 (5), 145-155.

Nattier, Jan. 2003. The Ten Epithets of the Buddha in the Translations of Zhi Qian 支謙. In: Annual Report of the International Research Institute for Advanced Buddhology 6, 207-250.

Özertural, Zekine. 2021. Alttürkische Handschriften Teil 4. Varia Buddhica: Buddhistische Gedichte und kleinere Sūtra-Texte. Stuttgart (VOHD 13,12).

Sanskrit-Wörterbuch der buddhistischen Texte aus den TurfanFunden und der kanonischen Literatur der SarvāstivādaSchule, begonnen von E. Waldschmidt, hrsg. von H. Bechert. Göttingen 1973 ff. 
Stönner, Heinrich. 1904. Zentralasiatische Sanskrittexte in Brāhmīschrift aus Idikutšahri, Chinesisch-Turkistan. I. Nebst Anhang: Uigurische Fragmente in Brāhmīschrift. SKPAW 44 (1904), 1282-1290.

Wilkens, Jens. 2001. Die drei Körper des Buddha (Trikāya). Das dritte Kapitel der uigurischen Fassung des Goldglanz-Sūtras (Altun Yaruk Sudur), Turnhout (Berliner Turfantexte 21).

Wilkens, Jens. 2007. Das Buch von der Sündentilgung. Edition des alttürkisch-buddhistischen Kšanti K1lguluk Nom Bitig. 1-2. Turnhout (Berliner Turfantexte 25).

Wilkens, Jens. 2021. Handwörterbuch des Altuigurischen. Göttingen.

Wille, Klaus. 2014. Survey of the Sanskrit Manuscripts in the Turfan Collection (Berlin). In: From Birch Bark to Digital Data: Recent Advances in Buddhist Manuscript Research Papers Presented at the Conference Indic Buddhist Manuscripts: The State of the Field Stanford, June 15-19 2009. Edited by Paul Harrison and Jens-Uwe Hartmann. Wien, 187-211.

Wille, Klaus. 2015. The Sanskrit Fragments Or.15015 in the Hoernle Collection. In: The British Library Sanskrit Fragments, vol. III, hrsg. von S. Karashima, J. Nagashima and K. Wille, Tokyo 2015 (Buddhist Manuscripts from Central Asia), 485-551.

Wille, Klaus. 1995. Verzeichnis der Orientalischen Handschriften in Deutschland Teil 7: Sanskrithandschriften aus den TurfanFunden: Die Katalognummern 1600-1799. Stuttgart.

Wille, Klaus. 2004. Verzeichnis der Orientalischen Handschriften in Deutschland Teil 9: Sanskrithandschriften aus den TurfanFunden: Die Katalognummern 2000 - 3199. Stuttgart. 
Wille, Klaus 2008. Verzeichnis der Orientalischen Handschriften in Deutschland Teil 10: Sanskrithandschriften aus den TurfanFunden: Die Katalognummern 3200-4362. Stuttgart.

Zieme, Peter. 2018. Kanișka in the Old Turkic Tradition. In: Great Journeys across the Pamir Mountains. A Festschrift in Honor of Zhang Guangda on his Eighty-Fifth Birthday. Leiden / Boston, 176-185. 